\title{
Estrés y afrontamiento en estudiantes de psicología
}

\author{
Mónica Cassaretto ${ }^{1}$, Cecilia Chau ${ }^{2}$, Haydeé Oblitas ${ }^{3}$ y Nancy Valdez ${ }^{4}$ \\ Pontificia Universidad Católica del Perú
}

\begin{abstract}
Se analizó la relación entre estrés, problemas más frecuentes y estilos de afrontamiento en 123 estudiantes universitarios de la especialidad de psicología de una universidad particular en Lima, mediante cuatro instrumentos: a) Ficha Demográfica (Cassaretto, Oblitas \& Valdez, 2000), b) Cuestionario de la Respuesta de Estrés (Valdez, 1999), c) Inventario sobre Estilos y Estrategias de Afrontamiento (Carver, Scheier \& Weintraub, 1989) y d) Cuestionario de Problemas (Seiffge-Krenke, 1995). Se consideraron las variables edad, sexo, trabajo, respuestas de estrés, problemas y estilos de afrontamiento. Los hallazgos indicaron que a mayor edad mayores niveles de estrés, y que estos eran más elevados al evaluarse los problemas relacionados con el futuro y el sí mismo; por otro lado, los estilos centrados en el problema y la emoción son los más utilizados en el afrontamiento y los que se relacionan con niveles de estrés más bajos.

Palabras clave: estrés, afrontamiento, problemas, estudiantes de psicología.
\end{abstract}

\section{Stress and coping among psychology students}

The relations among stress, problems and coping styles among 123 psychology students, in a private university in Lima, were analyzed. Four instruments were used: a) Demographic Sheet (Cassaretto, Oblitas \& Valdez, 2000), b) Stress Response Questionnaire (Valdez, 1999), c) Coping Inventory (Carver, Scheier \& Weintraub, 1989), d) Problem Questionnaire (SeiffgeKrenke, 1995). Variables as age, sex, job, stress responses, problems and coping styles were considered. The results showed that older people have higher stress levels, the problems related with future and self being the most stressful; finally, problem and emotion coping styles are related to lower levels of stress.

Key words: stress, coping, problems, psychology students.

Licenciada en Psicología Clínica. Profesora de la Especialidad de Psicología de la PUCP. Correo electrónico: mcassar@pucp.edu.pe.

2 Magister en Psicología. Profesora auxiliar de la Especialidad de Psicología de la PUCP. Correo electrónico: cchau@.pucp.edu.pe.

3 Licenciada en Psicología Clínica. Profesora de la Especialidad de Psicología de la PUCP. Correo electrónico: hoblita@pucp.edu.pe.

4 Licenciada en Psicología Clínica. Profesora de la Especialidad de Psicología de la PUCP. Correo electrónico: nvaldez@pucp.edu.pe. 

El estrés es un tema de gran interés en la actualidad, tanto en su estudio científico como en el marco de la vida cotidiana. Este hecho resulta comprensible si se toma en cuenta la manera en que afecta cada vez más al hombre de hoy en sus diversas realidades (Hobfoll, 1989).

El concepto de estrés se ha aplicado a diversos fenómenos psicosociales, lo que ha llevado a cierta confusión sobre el término. Sin embargo, puede considerarse como un estado producido por una serie de demandas inusuales o excesivas que amenazan el bienestar o integridad de una persona. En el intento de dominar dicha situación se corre el peligro de que los recursos de afrontamiento se vean superados, llevando a una perturbación en el funcionamiento del individuo, lo que podría causarle dolor emocional, enfermedad e incluso la muerte.

$\mathrm{Al}$ inicio de los estudios sobre estrés, se consideró al estrés como un estímulo, según el cual todos los acontecimientos negativos (como los desastres naturales, las condiciones nocivas para el organismo, los eventos traumatizantes, entre otros) eran asumidos como universalmente estresantes. Basado en este enfoque, Hans Selye (1936) consideró al estrés como un agente desencadenante, dado que es un elemento que atenta contra la homeostasis del organismo, que resulta en una respuesta fisiológica no específica; dicha respuesta era estereotipada e implicaba una activación del eje hipotalámico-hipofísico-suprarrenal y del sistema nervioso autónomo. A este proceso lo llamó el "Síndrome de Adaptación General", el que presenta tres etapas claramente diferenciadas: a) alarma, cuando el organismo percibe un agente que identifica como nocivo y genera una respuesta inicial de alerta, presentando diferentes síntomas y movilizando defensas para responder a la posible amenaza. Es una reacción intensa y no dura mucho; b) resistencia, dado que la primera 
fase no puede mantenerse por mucho tiempo se da lugar a la etapa en la cual el organismo busca adaptarse al agente nocivo (también denominado estresor) y desaparecen los síntomas iniciales. Aquí, hay un aumento del estado de alerta y mejora la capacidad de respuesta física; y c) agotamiento, si el estresor continúa, el organismo ingresa en la etapa donde reaparecen los síntomas y se produce una ruptura de los procesos de recuperación, siendo incluso posible que el proceso culmine con la muerte (Lazarus \& Folkman, 1986; Sandín, 1995).

Esta teoría, si bien es muy útil, adolece de una explicación de las diferencias individuales en las respuestas psicofísicas de estrés observadas en diversos sujetos ante los mismos acontecimientos estresantes. En este contexto, surge la teoría transaccional del estrés de Lazarus y Folkman (1986), la cual plantea que la persona y el ambiente mantienen una relación dinámica, mutuamente recíproca y bidireccional. Por ello, el estrés es considerado como un proceso que incluye las transacciones entre el individuo y su medio ambiente durante el cual la percepción de amenaza y/o daño ocasiona reacciones físicas y psicológicas. Dichas respuestas se caracterizan por ser de tipo rápido, intuitivo y automático, y son diferentes del pensamiento reflexivo, el cual es más lento y más abstracto (Lazarus \& Folkman, 1986; Taylor, 1999).

En el concepto actual de estrés, el proceso de valoración o evaluación psicológica despliega un papel importante. La evaluación puede ser de dos formas: i) Valoración primaria: es un patrón de respuesta inicial, en el cual la persona evalúa la situación en base a cuatro modalidades: a) valoración de amenaza, que se presenta cuando la persona anticipa un posible daño y/o peligro; b) valoración de desafío, se presenta ante una situación en la cual existe una valoración de amenaza pero además existe la posibilidad de ganancia, debido a la cual, la persona piensa que puede manejar el estresor satisfactoriamente por medio de sus recursos; c) evaluación de pérdida o daño, cuando ha habido algún perjuicio, como una lesión o enfermedad incapacitante, 
algún daño a la estima propia o social, o bien haber perdido algún ser querido; y d) valoración de beneficio, la cual no genera reacciones de estrés. ii) Valoración secundaria: se busca determinar las acciones a realizar para enfrentar el estresor y la percepción de los recursos o habilidades de afrontamiento. La interacción entre la valoración primaria y secundaria determina el grado de estrés, la intensidad y la calidad (contenido) de la respuesta emocional. Ambos son interdependientes (Lazarus \& Folkman, 1986).

Las reacciones de estrés se presentan agrupadas en tres bloques: fisiológicas, emocionales y cognitivas: a) fisiológicas, comprenden las respuestas neuroendocrinas y las asociadas al sistema nervioso autónomo como aumento de la presión sanguínea, incremento de la tasa cardiaca, incremento de la actividad estomacal e intestinal, dilatación de las pupilas, dolor de cabeza, incremento de la respiración, manos frías, sequedad de la boca, pies fríos, entre otras (Sandín, 1995). El efecto de esta respuesta sobre la salud a largo plazo puede ser fatal, ya que deteriora el sistema inmunológico, el cual es encargado de proteger a nuestro organismo de todo aquello que podría causarnos una enfermedad (Herbert, 1994); b) emocionales, abarcan las sensaciones subjetivas de malestar emocional como el temor, la ansiedad, la excitación, la cólera, la depresión, el miedo y la ira, principalmente (Sandín, 1995; Taylor, 1999). Las investigaciones muestran que los problemas de salud mental son generados, activados o exacerbados ante la exposición de eventos estresantes (Shepperd \& Kashani, 1991); c) cognitivas, existen tres tipos de respuestas cognitivas de estrés principales: la preocupación, la pérdida de control y la negación, las cuales se presentan acompañadas de bloqueos mentales, pérdida de memoria, sensación de irrealidad, procesos disociativos de la mente, entre otros (Sandín, 1995). Estos síntomas afectan el rendimiento de la persona y la relación que establezca con los demás.

El estrés posee diversas fuentes. Puede ser una demanda ambiental, social o interna que requiere que el individuo reajuste sus patrones de 
conducta (Holmes \& Rahe, 1967) (c.f. Sandín, 1995). Sin embargo, el grado de la reacción de estrés, más que depender de estos eventos dependerá del tipo de pensamientos evaluativos que la persona tenga; por ello, no siempre los eventos negativos ocasionarán malestar psicológico. El malestar se origina solamente cuando las demandas impuestas por la situación son percibidas o valoradas como excedentes a las habilidades para afrontarla (Folkman, Lazarus, Gruen \& De Longis, 1986).

Las fuentes generadoras de estrés pueden ser hechos positivos y negativos. Los estresores presentan como características básicas el ser intensos (capaces de producir un estado de sobrecarga que impide que podemos adaptarnos a ellos), provocar tendencias incompatibles (como las de aproximarnos y alejarnos de cierto objeto o actividad) y ser incontrolables (al ser evaluados como excedentes de nuestros recursos).

$\mathrm{La}$ adolescencia es una etapa que presenta diversas tareas que podrían convertirse en fuentes de estrés, tales como la búsqueda de identidad o la elección de futuro vocacional. Según la literatura revisada en diferentes países (Alemania, Estados Unidos, Perú) los adolescentes reportaron similares fuentes de estrés, tales como problemas vinculados a la escuela (la exigencia y dificultades académicas), déficit en habilidades sociales, incertidumbre respecto al futuro (desempleo, destrucción del medio ambiente y percepción de falta de éxito), dificultades familiares (alcoholismo, agresiones físicas, enfermedades, crisis económica, divorcio, falta de comunicación con los padres y entendimiento del rol de los padres), presión social (problemas de comunicación, deseo de ser aprobado y miedo al rechazo de los pares), problemas relacionados al uso del tiempo libre, problemas en las relaciones amorosas, y por último los problemas en relación a sí mismo (Castro de la Mata \& Rojas, 1998; Martínez \& Morote, 2001; Montenegro, 2001; Phelan, Yu \& Davidson, 1994 (c.f. Trahtemberg, 1995); Seiffge-Krenke, 1995, 1998). 
Junto con estas semejanzas, se encuentra una diferencia saltante entre los adolescentes y jóvenes peruanos asociados a las fuentes de estrés; ésta es la presencia de la violencia callejera y los problemas económicos aunados a la pobreza (Castro de la Mata \& Rojas, 1998).

Las consecuencias negativas del estrés son reguladas o mediatizadas por un proceso denominado afrontamiento, el que es considerado como un proceso dinámico, en respuesta a demandas objetivas y a evaluaciones subjetivas de la situación. Para Lazarus y Folkman (1986) el afrontamiento no es un estilo de personalidad constante sino que, por el contrario, está formado por ciertas cogniciones y conductas que se ejecutan en respuesta a situaciones estresantes específicas. La relación entre estrés y afrontamiento es recíproca, debido a que las acciones que realiza una persona para afrontar un problema afectan la valoración del problema y el subsecuente afrontamiento (Lazarus \& Folkman, 1986; Stone, Greenberg, Kennedy-Moore \& Newman, 1991).

En general, el afrontamiento ayuda a identificar los elementos que intervienen en la relación dada entre situaciones de vida estresantes y síntomas de enfermedad (Kobasa, Maddi \& Kahn, 1982). El afrontamiento actúa como regulador de la perturbación emocional; si es efectivo no se presentará dicho malestar; en caso contrario, podría verse afectada la salud de forma negativa, aumentando así el riesgo de mortalidad y morbilidad. El afrontamiento puede ser entendido entonces como el conjunto de esfuerzos cognitivos y conductuales orientados a manejar (reducir, minimizar, dominar o tolerar) las demandas internas y externas de una determinada situación estresante.

El estudio del afrontamiento ha llevado a conceptualizar distintos tipos de dimensiones dentro de la personalidad; a éstas se les denomina estilos y estrategias. Los estilos de afrontamiento son aquellas predisposiciones personales para hacer frente a diversas situaciones y son los que determinarán el uso de ciertas estrategias de afrontamiento, así como su estabilidad temporal y situacional. Por otro lado, las estrategias de afrontamiento son los procesos concretos y 
específicos que se utilizan en cada contexto y pueden ser altamente cambiantes dependiendo de las condiciones desencadenantes (Carver \& Scheier, 1994; Fernández-Abascal, Palmero, Chóliz \& Martínez, 1997).

Lazarus y Folkman (1986) plantean dos estilos de afrontamiento: a) estilos centrados en el problema, y b) estilos centrados en la emoción. Carver, Scheier y Weintraub (1989) señalan que ambos estilos son importantes, pero consideran que el proceso de afrontamiento es mucho más complejo, presentando quince estrategias: el afrontamiento activo, la planificación, la supresión de actividades competitivas, la postergación del afrontamiento, el soporte social instrumental y emocional, la reinterpretación positiva, la aceptación, la negación, el acudir a la religión, la expresión de las emociones, el uso de alcohol y drogas y el no compromiso conductual y cognitivo. Estas respuestas de afrontamiento brindan una medida más precisa que las planteadas inicialmente por Folkman y Lazarus (1986).

En la literatura reciente sobre estrés y afrontamiento se mencionan dos orientaciones básicas para afrontar el estrés: la aproximación (lucha) y la evitación (huida). El afrontamiento aproximativo incluye todas aquellas estrategias de confrontación y enfrentamiento al problema que estan generando distrés o una emoción negativa, mientras que el afrontamiento evitativo incluye todas aquellas estrategias cognitivas o comportamentales que permiten escapar de, o evitar el problema o las emociones negativas. Ambas estrategias no son excluyentes, es decir, las conductas no podrían clasificarse como aproximativas o evitativas absolutas (Rodríguez, 1995).

Una serie de investigaciones llevadas a cabo tanto por Frydenberg y colegas, así como por otros autores en este campo de estudio (Endler \& Parker, 1994; Frydenberg, 1997; Frydenberg \& Lewis, 1999; Patterson \& McCubbin, 1987; Phelps \& Jarvis, 1994) plantean la existencia de diferencias en el afrontamiento según género. Estas diferencias no sólo se presentan a nivel de las estrategias, sino además 
en los estilos de afrontamiento. Tanto hombres como mujeres difieren en la forma como manejan el estrés; éstas diferencias se han encontrado en la construcción y utilización de las redes sociales (Belle, 1987; Butler \& Giordano,1985) (c.f. Wiebe, 1991), en la respuesta de afrontamiento (Miller \& Kirsch, 1987) (c.f. Wiebe, 1991) y en el proceso cognitivo adaptativo frente a dichas situaciones estresantes (Smith, O'Keefe \& Jenkins, 1988) (c.f. Wiebe, 1991). Patterson y McCubbin (1987) añaden que las diferencias entre hombres y mujeres respecto al soporte social reflejarían diferencias en la socialización, enfatizándose en las mujeres más que en los hombres conductas de afiliación.

Lepose, Erans y Schneider (1991) refieren que el soporte social puede disminuir el efecto de ciertas circunstancias estresantes dado que la percepción o creencia de que existen otras personas disponibles para promover el confort emocional o asistencia práctica cuando uno se encuentra frente a determinados eventos, es positiva. El soporte social es un amortiguador de los eventos negativos.

Varios autores coinciden en reconocer la influencia de los cambios en el desarrollo en la naturaleza del afrontamiento (Frydenberg \& Lewis, 1993, 1997, 1999; Kavsek \& Seiffge-Krenke, 1996). La edad es un factor importante que influye en el afrontamiento adolescente; sin embargo, las investigaciones orientadas a estudiar las diferencias según la edad han sido escasas si las comparamos con las realizadas en relación al género. Seiffge-Krenke (1995) postula que la mayor parte de cambios relacionados con la edad ocurren en el llamado afrontamiento interno, que tiene que ver con conductas que buscan resolver el problema. El uso regular de este estilo se da en los adolescentes de las etapas media y tardía, sobre todo en aquellos que tienen problemas relacionados con los padres, estudios y con el sí mismo. Además, la autora agrega que en los adolescentes mayores, a diferencia de los adolescentes más jóvenes, se produce generalmente un incremento en el estilo de afrontamiento activo, especialmente en los problemas vinculados con la escuela, el futuro, los padres, las relaciones románticas y el sí mismo. 
A pesar de que el tema del estrés está muy difundido entre las personas, pocos son los estudios en nuestro medio que se han encargado de identificar sus principales características. Esto se observa también entre los estudiantes universitarios, quienes especialmente atraviesan por una serie de cambios y demandas propias de la vida académica y personal.

Además, la ocupación diaria de las personas produce estrés en mayor o menor grado. Diversas investigaciones indican como más estresantes las profesiones de ayuda; esto debido al enfrentamiento entre la filosofía humanista de estos profesionales y el sistema deshumanizado imperante (Moreno, Oliver \& Aragoneses, 1991). Dentro de las profesiones de ayuda y de relación directa con las personas se encuentran principalmente la educación, trabajo social, psicología, psiquiatría, medicina y enfermería.

Si las profesiones de ayuda están consideradas entre las más estresantes y los profesionales de la salud ven potenciado el efecto del estrés, la posibilidad de que estas personas vean limitadas sus capacidades intelectuales y emocionales se incrementa. En ese sentido, los estudiantes de psicología pueden verse doblemente afectados debido a que aún no cuentan con todos los recursos personales y sociales, como sí los tienen los profesionales con mayor experiencia. Por este motivo surge el interés de conocer el nivel y fuentes de estrés, así como los estilos/estrategias de afrontamiento más utilizados por un grupo de estudiantes de psicología de una universidad privada en Lima.

\section{Metodología}

Esta es una investigación ex post facto, de tipo correlacional, donde se busca determinar la relación entre problemas más comunes, estilos y estrategias de afrontamiento y niveles de estrés en un grupo de estudiantes de psicología (Hernández, Fernández \& Baptista, 1996). 
Estrés y afrontamiento en estudiantes de psicología

\section{Participantes}

La muestra del estudio estuvo conformada por 123 estudiantes de psicología de los primeros años de formación, de una universidad privada de la ciudad de Lima. La distribución por sexo indicó mayor número de estudiantes de sexo femenino (76\%) que masculino $(24 \%)$, tal y como se observa en la Figura 1.

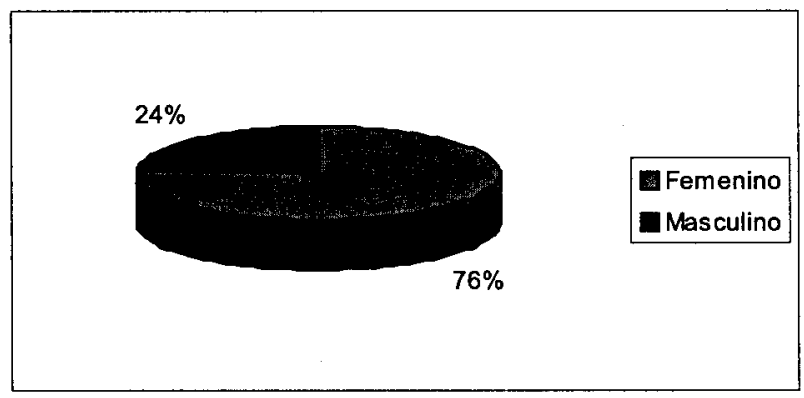

Figura 1. Distribución por sexo.

En cuanto a la edad de los participantes, se observa en la Figura 2 una preponderancia de aquellos cuyas edades fluctuaron entre los 21 y los 23 años (69\%), seguidos por aquellos que tenían entre 19 y 20 años (24\%); mientras que en menor proporción se encontraron los que cuentan entre 24 y 25 años $(7 \%)$ de edad.

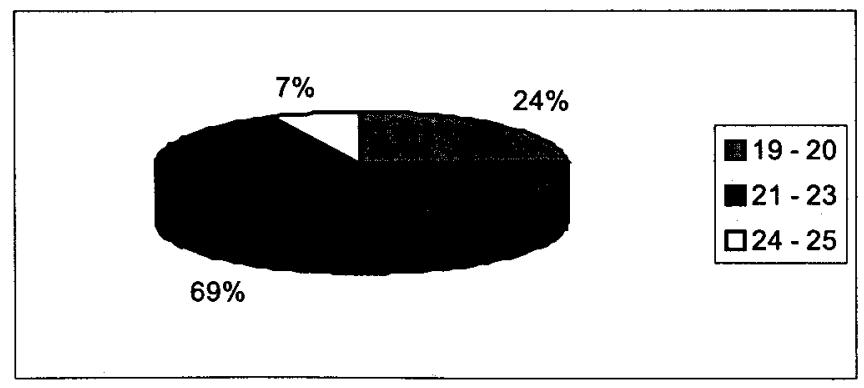

Figura 2. Distribución por edad. 
La distribución de los estudiantes según la especialidad elegida indica que la mayoría de ellos prefirió la Psicología Clínica (61\%), seguidos por los que prefirieron la Social $(26 \%)$ y finalmente por los que eligieron el campo Educacional (9\%) según lo muestra la Figura 3.

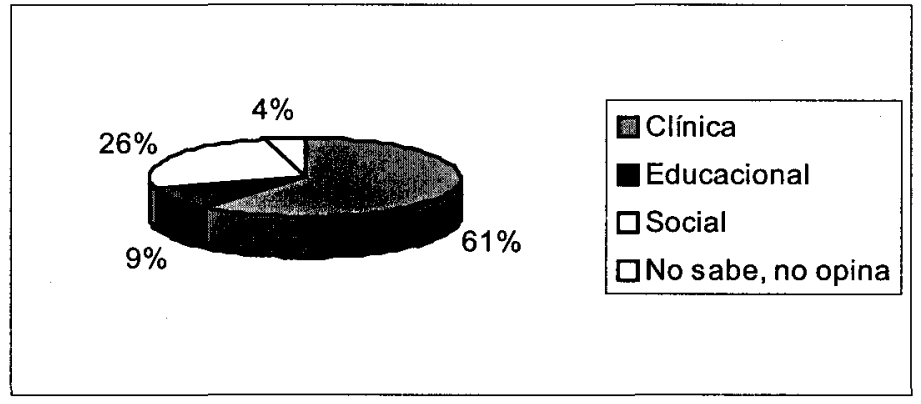

Figura 3. Distribución por especialidad.

\section{Instrumentos}

Se utilizaron cuatro instrumentos: a) Ficha Demográfica (Cassaretto, Oblitas \& Valdez, 2000), b) Cuestionario de la Respuesta de Estrés (Valdez, 1999), c) Inventario sobre Estilos y Estrategias de Afrontamiento (Carver, Scheier \& Weintraub, 1989) y d) Cuestionario de Problemas (Seiffge-Krenke, 1995).

a) Ficha Demográfica: la Ficha Demográfica fue construida para fines de este estudio y evaluó los siguientes datos sociodemográficos sobre la muestra de estudio: sexo, edad, especialidad que piensa cursar (clínica, social o educacional), realiza o no otros estudios, trabajo y deportes.

b) Cuestionario de la Respuesta de Estrés: este cuestionario elaborado por Valdez (1999) consta de 33 ítems y analiza tres componentes de la respuesta de estrés: a) emocional, b) fisiológico y c) cognitivo. La validez del instrumento se obtuvo mediante criterio de 
jueces, con índices de acuerdo mayores a 0.80 . La confiabilidad por áreas mostró alfas de Cronbach mayores a 0.75 y correlaciones ítemtest mayores de 0.37. La prueba total arrojó un alfa de 0.91 .

c) Cuestionario de Estilos de Afrontamiento COPE (Coping Estimation): este instrumento fue desarrollado por Carver, Scheier y Weintraub (1989) en su versión disposicional, la que fue construida siguiendo dos modelos teóricos: a) Modelo de Lazarus, b) Modelo de Autoregulación Conductual de Bandura. Este inventario tiene 52 ítems, con un sistema de respuesta en formato de tipo Likert de cuatro puntos de calificación, consta de 13 escalas (afrontamiento activo, planificación, supresión de actividades competentes, postergación del afrontamiento, búsqueda de apoyo social por razones instrumentales, búsqueda de apoyo social por razones emocionales, reinterpretación positiva y crecimiento, aceptación, negación, acudir a la religión, enfocar y liberar emociones, desentendimiento conductual y desentendimiento mental) que miden estrategias de afrontamiento y se organizan alrededor de tres estilos de afrontamiento planteados por los autores: a) centrado en el problema, b) centrado en la emoción y c) otros estilos. Este inventario fue adaptado para el Perú en 1996 por Casuso y ha demostrado su validez y confiabilidad en múltiples investigaciones (Chau, 1999; Rojas, 1997).

d) Cuestionario de Problemas: este cuestionario fue construido por Seiffge-Krenke (1995) y consta de 64 ítems sobre problemas cotidianos agrupados en siete situaciones: a) en relación al colegio, b) al futuro, c) a los padres, d) a los pares, e) al tiempo libre, f) a las relaciones amorosas y g) al sí mismo. Cada ítem es categorizado según una escala Likert de 5 puntos que va desde 1 (nada estresante) hasta el 5 (muy estresante), permitiendo medir el grado de estrés. SeiffgeKrenke estudió la validez de constructo de la prueba y realizó el análisis factorial utilizando el método de componentes principales y la rotación Varimax. Luego de los análisis correspondientes, encontró que la estructura de siete factores era la más adecuada pues de este modo 
se explicó el $69 \%$ de la varianza total. En relación a la confiabilidad, en el análisis de cada factor se encontraron Coeficientes Alfa de Cronbach que oscilaron entre .70 y .84 , y correlaciones ítem-test corregidas que fluctuaron entre .29 y .52 , cumpliendo en ambos casos los criterios para ser considerados adecuados. Posteriormente, Chau (2003) realizó la adaptación de la prueba en el Perú con estudiantes universitarios encontrando alta validez y confiabilidad en la versión de seis escalas que se utilizó en ésta investigación.

\section{Resultados}

Según se observa en el Cuadro 1, los problemas que generaron mayores niveles de estrés en los participantes fueron aquellos relacionados con el futuro y las preocupaciones por el sí mismo, mientras que las relaciones románticas generaron menor malestar emocional.

\section{Cuadro 1}

Tipos de problema más frecuentes

\begin{tabular}{|l|cl|}
\hline Tipo de Problema & $M$ & $D E$ \\
\hline Universidad & 2,1 & 0,7 \\
Futuro & 2,7 & 0,8 \\
Padres & 2,1 & 0,8 \\
Amigos & 2,1 & 0,7 \\
Relaciones románticas & 2,0 & 0,8 \\
Sí mismo & 2,2 & 0,7 \\
Puntaje Global & 2,2 & 0,6 \\
\hline
\end{tabular}

$n=123$

En cuanto a las diferencias por sexo en el Cuestionario de Problemas, el Cuadro 2 muestra que las mujeres experimentaron mayores niveles de estrés que los varones en las escalas relacionadas con los padres, las relaciones románticas y con los amigos, respectivamente. 
Estrés y afrontamiento en estudiantes de psicología

\section{Cuadro 2}

Diferencias en escalas del Cuestionario de Problemas de acuerdo a sexo

\begin{tabular}{|c|c|c|c|c|c|}
\hline \multirow[t]{2}{*}{$\begin{array}{l}\text { Tipo de } \\
\text { Problema }\end{array}$} & \multirow[t]{2}{*}{$Z$} & \multicolumn{2}{|c|}{ Masculino } & \multicolumn{2}{|c|}{ Femenino } \\
\hline & & $M$ & $D E$ & $M$ & $D E$ \\
\hline Padres & 2,22 & 2,2 & 0,8 & 1,8 & 0,9 \\
\hline Relaciones Románticas & 2,09 & 2,2 & 0,7 & 1,9 & 0,9 \\
\hline Amigos & 2,18 & 2,1 & 0,8 & 1,8 & 0,8 \\
\hline
\end{tabular}

$p<.05$ dos colas

Al comparar los principales problemas generadores de estrés de acuerdo a la especialidad elegida, utilizando un estadístico de comparación multivariado (LSD), se encontró que los estudiantes inscritos en la especialidad Educacional manifestaron mayor nivel de estrés ante los problemas con los padres y los amigos, mientras que los estudiantes de la especialidad Clínica indicaron sentirse menos afectados por los problemas relacionados con sus padres y los amigos, tal y como se observa en el Cuadro 3.

\section{Cuadro 3}

Diferencias en escalas del Cuestionario de Problemas de acuerdo a especialidades

\begin{tabular}{|l|cccccc|}
\hline & \multicolumn{2}{|c}{ Clínica } & \multicolumn{2}{c|}{ Educacional } & \multicolumn{2}{c|}{ Social } \\
\hline & $M$ & $D E$ & $M$ & $D E$ & $M$ & $D E$ \\
Padres & 2,00 & 0,70 & 2,50 & 0,90 & 2,20 & 0,90 \\
Amigos & 2,00 & 0,70 & 2,50 & 0,70 & 2,30 & 0,70 \\
\hline
\end{tabular}

$p<.05$

En relación con el afrontamiento, las estrategias más utilizadas por los participantes de la muestra fueron la reinterpretación positiva, la búsqueda de apoyo social emocional y la planificación; mientras que las que se utilizaron con menor frecuencia fueron la negación, el desentendimiento conductual y acudir a la religión. Asimismo, entre 
los estilos de afrontamiento primaron los centrados en el problema, seguidos cercanamente por los centrados en la emoción, mientras que los otros estilos fueron poco utilizados (ver Cuadro 4).

Se puede observar en el Cuadro 5 que en relación con las diferencias de afrontamiento según sexo, trabajo y deporte, las mujeres acudieron a la religión y se centraron más en la emoción que los varones ante las situaciones de estrés. Aquellos que trabajaban utilizaron una estrategia de afrontamiento más activo y liberaron con mayor facilidad sus emociones. Finalmente, los participantes que no practicaban deporte reportaron una mayor utilización de la negación como estrategia de afrontamiento ante situaciones difíciles.

\section{Cuadro 4}

Medias y Desviaciones Estándar de las Estrategias y Estilos de Afrontamiento del COPE

\begin{tabular}{|l|cc|}
\hline Estrategias de Afrontamiento & $M$ & $D E$ \\
\hline Activo & 9,8 & 2,1 \\
Planificación & 11,1 & 2,7 \\
Supresión de actividades competentes & 8,4 & 2,4 \\
Postergación & 8,9 & 2,2 \\
Apoyo social instrumental & 10,6 & 3,0 \\
Apoyo social emocional & 11,1 & 3,1 \\
Reinterpretación positiva & 11,4 & 2,8 \\
Aceptación & 10,2 & 2,5 \\
Acudir a la religión & 7,6 & 3,8 \\
Negación & 5,0 & 1,4 \\
Liberación & 8,7 & 2,3 \\
Desentendimiento conductual & 5,6 & 1,6 \\
Desentendimiento mental & 7,8 & 2,2 \\
Estilos de Afrontamiento & & 8,1 \\
Centrado en el problema & 48,8 & 7,1 \\
Centrado en la emoción & 45,3 & 4,3 \\
Otros estilos & 22,1 & \\
\hline
\end{tabular}

$n=123$ 
Estrés y afrontamiento en estudiantes de psicología

\section{Cuadro 5}

Diferencias en el afrontamiento de acuerdo a sexo, trabajo y deporte

\begin{tabular}{|l|ccccccccc|}
\hline Afrontamiento & \multicolumn{3}{|c}{ Sexo } & \multicolumn{3}{c|}{ Trabajo } & \multicolumn{3}{c|}{ Deporte } \\
\hline & $Z$ & $\mathrm{~F}$ & $\mathrm{M}$ & $Z$ & $\mathrm{Si}$ & No & $Z$ & $\mathrm{Si}$ & No \\
Acudir a la religión & $2,38 *$ & $8,15 *$ & $6,24 *$ & $-1,17$ & 6,88 & 7,93 & $-1,50$ & 8,15 & 6,90 \\
Activo & 0,89 & 9,91 & 9,52 & $2,12 *$ & $10,72 *$ & $9,53 *$ & $-1,70$ & 9,94 & 9,17 \\
Liberación & 0,90 & 8,81 & 8,38 & $2,13 *$ & $9,52 *$ & $8,45 *$ & 0,06 & 8,70 & 8,72 \\
Negación & 0,93 & 5,08 & 4,79 & 0,27 & 5,16 & 5,07 & $2,21 *$ & $4,80 *$ & $5,66 *$ \\
C. Emoción & $2,08 *$ & $46,21 *$ & $43,10 *$ & 0,81 & 46,52 & 45,17 & $-0,70$ & 45,94 & 44,83 \\
\hline
\end{tabular}

$p<.05$ dos colas $\quad M=$ masculino, $F=$ femenino

Al analizar las diferencias en el afrontamiento de acuerdo a las especialidades, observamos en el Cuadro 6 que los estudiantes de la especialidad Educacional acudieron con mayor frecuencia a la religión ante situaciones estresantes y, además, tendieron a reprimir sus emociones. Por otro lado, los estudiantes de la especialidad Clínica liberaron con mayor frecuencia sus emociones y acudieron menos a la religión ante sus dificultades.

\section{Cuadro 6}

Diferencias en el afrontamiento de acuerdo a especialidades

\begin{tabular}{|l|cccccc|}
\hline Afrontamiento & \multicolumn{2}{|c}{ Clínica } & \multicolumn{2}{c|}{ Educacional } & \multicolumn{2}{c|}{ Social } \\
\hline & $M$ & $D E$ & $M$ & $D E$ & $M$ & $D E$ \\
Acudir a la religión & 6,90 & 3,40 & 12,00 & 3,40 & 8,10 & 3,90 \\
Liberación & 9,10 & 2,20 & 7,50 & 2,30 & 8,30 & 2,00 \\
\hline
\end{tabular}

Aún-cuando se realizaron comparaciones estadísticas, los participantes del estudio no mostraron diferencias significativas en cuanto a reacciones de estrés de acuerdo a sexo, edad, especialidad, trabajo o práctica de deporte.

Al correlacionar las reaccionès de estrés y el tipo de afrontamiento (Cuadro 7), se encontró que los mayores niveles de reacción se 
correlacionaron con una mayor utilización de otros estilos y con las estrategias de búsqueda de apoyo social emocional, liberación de emociones y desentendimiento mental. Sin embargo, a mayores niveles de estrés se encontró menor utilización de las estrategias de afrontamiento denominadas supresión de actividades competentes y reinterpretación positiva.

\section{Cuadro 7}

Correlación entre afrontamiento y reacción de estrés

\begin{tabular}{|l|cccc|}
\hline Afrontamiento & Cognitiva & Emocional & Fisiológica & Global \\
\hline Supresión de actividades competentes & $-0,22 *$ & $-0,29 *$ & $-0,27^{*}$ & $-0,28^{*}$ \\
Apoyo social emocional & 0,18 & $\mathbf{0 , 2 1 *}$ & $0,18^{*}$ & $0,20^{*}$ \\
Reinterpretación positiva & $-0,11$ & $-0,22^{*}$ & $-0,05$ & $-0,13$ \\
Liberación & 0,13 & $0,29^{*}$ & 0,12 & $0,20^{*}$ \\
Desentendimiento mental & $0,22 *$ & $0,23^{*}$ & 0,11 & $0,19 *$ \\
Otros estilos & 0,21 & 0,32 & 0,10 & $0,22 *$ \\
\hline
\end{tabular}

$* p<.05$

En relación con los tipos de problemas generadores de estrés, aquellos relacionados con el futuro y la universidad fueron los que ocasionaron reacciones de estrés agudo más intensas (ver Cuadro 8).

\section{Cuadro 8}

Correlación entre reacción de estrés y Cuestionario de Problemas

\begin{tabular}{|l|cc|}
\hline Reacciones de Estrés & Universidad & Futuro \\
\hline Cognitiva & $0,21^{*}$ & $0,26^{*}$ \\
Emocional & 0,16 & $0,25^{*}$ \\
Fisiológica & 0,18 & 0,15 \\
Global & $0,19 *$ & $0,23 *$ \\
\hline
\end{tabular}

$* p<.05$

En el Cuadro 9 se observa que ante la presencia de problemas relacionados con la universidad, las estrategias más utilizadas fueron 
la planificación y el desentendimiento mental; mientras que los denominados otros estilos fueron los más reportados en el afrontamiento. Frente al futuro, los otros estilos continuaron siendo los más utilizados junto con el desentendimiento mental como estrategia. Cuando se produjeron problemas con los amigos, los participantes del estudio nuevamente optaron por los otros estilos para el afronte y utilizaron el desentendimiento mental y el conductual como estrategias para hacerles frente. Asimismo, en relación con los problemas amorosos, estos correlacionaron con la estrategia denominada liberación de emociones. Finalmente, si las preocupaciones se relacionaron con el sí mismo, los participantes del estudio utilizaron más los otros estilos de afrontamiento y el desentendimiento conductual, la negación, el desentendimiento mental y la liberación como estrategias, dejando de lado la reinterpretación positiva.

\section{Cuadro 9}

Correlación entre afrontamiento y cuestionario de problemas

\begin{tabular}{|c|c|c|c|c|c|c|}
\hline Afrontamiento & Universidad & Futuro & Amigos & Romance & $\begin{array}{c}\text { Sí } \\
\text { mismo }\end{array}$ & Global \\
\hline $\begin{array}{l}\text { Planificación } \\
\text { Reinterpretación }\end{array}$ & $0,20 *$ & 0,13 & 0,00 & 0,10 & 0,06 & 0,12 \\
\hline Positiva & $-0,07$ & $-0,16$ & $-0,08$ & $-0,03$ & $-0,22 *$ & $-0,12$ \\
\hline Negación & 0,13 & 0,18 & 0,15 & 0,13 & $0,24 *$ & $0,19 *$ \\
\hline $\begin{array}{l}\text { Liberación } \\
\text { Desentendimiento }\end{array}$ & 0,07 & 0,16 & 0,07 & $0,21 *$ & $0,20 *$ & 0,16 \\
\hline $\begin{array}{l}\text { Conductual } \\
\text { Desentendimiento }\end{array}$ & 0,08 & 0,07 & $0,20 *$ & 0,05 & $0,25 *$ & 0,15 \\
\hline Mental & $0,24 *$ & $0,25 *$ & $0,30 *$ & 0,11 & $0,20 *$ & $0,26 *$ \\
\hline Otros estilos & $0,20 *$ & $0,24 *$ & $0,27 *$ & 0,19 & $0,31 *$ & $0,28 *$ \\
\hline
\end{tabular}

$* p<.05$

En el Cuadro 10 se aprecia que en los participantes del estudio, a mayor edad se presentó mayor preocupación por el futuro, se produjeron menores reacciones de estrés cognitivo, emocional, y global, y se utilizó con mayor frecuencia la negación como estrategia de afrontamiento. 


\section{Cuadro 10}

Relación entre las variables de estudio y la edad

\begin{tabular}{|cccccc|}
\hline & Futuro & R. Cognitiva & R.Emocional & R. Global & Negación \\
\hline$R$ & 0,23 & $-0,25$ & $-0,20$ & $-0,22$ & 0,20 \\
\hline
\end{tabular}

$p<.05$ dos colas

Para obtener mayor profundidad en la comprensión de los hallazgos, se realizó un análisis de regresión múltiple escalonado con el fin de determinar las principales variables que determinan los niveles de estrés, encontrándose que éstas pueden ser explicadas en un $20 \%$ por tres variables $(\mathrm{R}=0.44, \mathrm{R}$ cuadrado ajustado $=0.17$, $\mathrm{F}(3,106)=8.63, \mathrm{p}<0.01)$. El nivel de estrés está determinado por el grado de preocupación por el futuro, la edad y la utilización de la estrategia denominada supresión de actividades competentes (ver Cuadro 11).

\section{Cuadro 11}

Análisis de Regresión Múltiple: variables de investigación y niveles de estrés

\begin{tabular}{|lrrrl|}
\hline Variables Independientes & B & Beta & t & Sig. \\
\hline Edad & $-5,15$ & $-0,27$ & $-3,05$ & 0,00 \\
Preocupación por futuro & 8,05 & 0,29 & 3,27 & 0,00 \\
Supresión de actividades competentes & $-2,29$ & $-0,26$ & $-2,93$ & 0,00 \\
\hline
\end{tabular}

\section{Discusión}

Al contrastar la teoría revisada y los resultados del estudio, encontramos que la muestra estuvo conformada por participantes muy jóvenes (menores de 23 años), la mayoría de ellos fueron mujeres y se orientan al área de psicología clínica, seguidos por los del área social. Esto reflejaría la continuación del estereotipo asociado a ciertas carreras en Latinoamérica, donde aún profesiones como la psicología albergan población principalmente femenina 
debido a que continúa la visión de que las mujeres son más empáticas y sensibles, con una notoria preferencia por la especialidad clínica. Así Ragúz (1995), quien evaluó las percepciones sobre estereotipos femenino y masculino, encontró que la percepción de lo femenino se asociaba a la afectividad y expresividad de sentimientos, lo que llevaría a que sea más factible que las mujeres elijan dicha carrera en comparación con los varones.

Respecto a los niveles de estrés, se observó que éstos son moderados, lo que podría deberse a que la aplicación de las pruebas se realizó en la cuarta semana de clases, periodo en el que los alumnos todavía no estaban sobrecargados con trabajos y presiones académicas. Sin embargo, estos niveles no podrían considerarse del todo benignos y sería necesario un seguimiento a lo largo del semestre académico para ver cómo ellos podrían haber variado. Llama la atención que al analizar por componentes la respuesta de estrés, se observó que los indicadores emocionales fueron más altos, es decir, los alumnos serían más conscientes de sus señales de estrés a partir de sus respuestas afectivas. Esto podría deberse a que la muestra estuvo conformada por estudiantes de psicología, los cuales por definición son más sensibles ante sus propias emociones, a las que suelen darles la relevancia del caso.

Sin embargo, observamos que a pesar de ello no reconocieron con tanta facilidad los componentes cognitivos de este fenómeno. Esto podría llevar a que las campañas de manejo del estrés en esta población den más énfasis en los componentes afectivos del mismo, para que así se logre una adecuada identificación y pueda prevenirse la presencia de indicadores de estrés más crónicos, los cuales afectan definitivamente la salud de las personas.

En relación a las principales preocupaciones, tomando en cuenta las condiciones socioeconómicas del país y la etapa del desarrollo en la que estos jóvenes se encuentran, en donde el logro personal y por 
ende el social depende mucho de la realización profesional, es esperable que el futuro y el sí mismo causen mayores niveles de estrés (Papalia, 1992). La incertidumbre, la novedad y la inestabilidad con la que estos temas se propician en el Perú, incrementan los niveles del mismo, que per se suelen producirse. Una adecuada elección hoy podría ser absolutamente errónea en un futuro no muy lejano, donde el mercado laboral podría cerrarse aún cuando en la elección de la especialidad también se hayan considerado los aspectos económicos.

Asimismo, es importante reflexionar acerca de la carrera de psicología, la que no sólo es vista como una profesión, sino también como una guía para el autoconocimiento y la resolución de conflictos personales, por lo que las preocupaciones sobre el sí mismo se hacen más patentes en este tipo de muestra en la medida que van profundizando en sus estudios.

En términos de las estrategias de afrontamiento, se encontró que la muestra utiliza con mayor frecuencia las estrategias de reinterpretación positiva, búsqueda de soporte social por motivos emocionales y planificación. Mientras que las estrategias menos usadas son la negación, el desentendimiento conductual y acudir a la religión. Esto nos lleva a pensar que dichos alumnos utilizan las estrategias más saludables y adaptativas, ya que tienden a ser más activas, mientras que las segundas son estrategias más pasivas. Esto es similar a lo encontrado por Martínez y Morote (2001), quienes reportaron en adolescentes entre los 13 y los 18 años la predilección por estrategias más efectivas para el afrontamiento del estrés, tales como esforzarse, tener éxito y concentrarse en resolver el problema. Este hallazgo es similar al de Casuso (1999) quien, al investigar estudiantes universitarios, encontró que la muestra total presentó una mayor preferencia por los estilos de planificación, postergación del afrontamiento y afrontamiento activo.

La utilización de la reinterpretación positiva y planificación como estrategias también podría explicarse en términos de lo que 
ellos consideran como esperable en un futuro psicólogo y/o de las habilidades y recursos propios con los que cuentan para ejercer la profesión. Aquello que consideren más adecuado en el ejercicio de su profesión y la imagen profesional que deseen dar podría intervenir en la elección y reporte de una estrategia considerada adaptativa y la desvalorización de aquellas consideradas como desadaptativas.

Asimismo, encontramos altos niveles de búsqueda de soporte social, ya sea por motivos instrumentales y/o emocionales, lo que refleja el reconocimiento de la existencia de redes sociales de apoyo. El hecho de que el soporte sea de ambas modalidades se convertiría en un fuerte recurso de afrontamiento, ya que se sabe que la pérdida de soporte social durante un tiempo crítico puede ser muy estresante, especialmente para las personas con elevadas necesidades de ello, pero con pocas oportunidades para obtenerlo (Taylor, 1999).

La búsqueda de soporte emocional también guarda correspondencia con la etapa de desarrollo y la identificación de género. Tomando en cuenta que los participantes del estudio son jóvenes, la búsqueda de apoyo emocional es una forma usual en el afronte de problemas (Papalia, 1992). En esta etapa del desarrollo y quizás debido a las características propias de la muestra (estudiantes de psicología) podrían encontrar satisfacción en el soporte brindado por sus compañeros (sería interesante saber quiénes son los que brindan este apoyo emocional) al considerarlos como más comprensivos y menos críticos que su familia, que es otra fuente importante de soporte social. Además, debido a que la mayor parte de la muestra está conformada por mujeres, según Russell, Booth, Reed y Laughlin (1997) es frecuente que ellas tiendan a buscar soporte social en otras mujeres con las que se puedan identificar según género, estudio y condiciones de vida parecidas. Esto también podría explicar la elección de un estilo más centrado en la emoción.

Por otro lado, es importante observar que la negación y el desentendimiento conductual fueron las estrategias menos utilizadas, 
ya que éstas, si bien generan un alivio temporal de las reacciones de estrés, a largo plazo podrían acarrear problemas futuros.

En cuanto a los estilos de afrontamiento, encontramos que éstos son mixtos y básicamente centrados en el problema y la emoción. En ese sentido, se utiliza con similar proporción aquellos estilos centrados en el problema así como los centrados en la emoción y en menor grado se utilizó el último estilo, al que Carver y Scheier (1994) denominan provisionalmente "otros estilos". En todo caso, recordemos que otros estudios han observado que el uso de las estrategias de afrontamiento focalizadas en el problema está relacionado con un mayor bienestar físico. Por el contrario, aquellos sujetos que preferentemente utilizan estrategias paliativas, como es el centrarse en las emociones o el escape conductual y/o cognitivo, manifiestan un mayor malestar físico (Carver \& Scheier, 1994). Por ello, las mujeres participantes de la investigación usarían estilos extremadamente útiles para enfrentar el estrés.

$\mathrm{Al}$ analizar los resultados relacionando variables se encontró una asociación significativa entre sexo y el uso del estilo de afrontamiento. Así, las mujeres son las que más utilizan el estilo centrado en las emociones, hallazgo que guarda relación con la literatura peruana (Canessa, 2000; Casuso, 1999; Chau, 1999; Martínez \& Morote, 2001), al igual que la utilización del afrontamiento religioso.

Cuando se analizaron los problemas generadores de estrés con las estrategias de afrontamiento, se observó que aquellos problemas vinculados con la universidad guardan relación con la planificación y el desentendimiento mental. La utilización de la planificación podría entenderse en términos de lo esperable en función de las propias expectativas profesionales. Sin embargo, considerando la etapa del desarrollo en la que se encuentran los participantes de la muestra, el desentendimiento mental podría resultarles más adaptativo en función del cumplimiento con otros problemas que tengan que resolver y con 
la forma como tienden a sobrevalorar sus habilidades y recursos para resolver los mișmos.

Frente al futuro, los otros estilos fueron los más utilizados, principalmente la estrategia denominada desentendimiento mental. En este caso, sería más bien la realidad social y económica del país la que estaría superando las habilidades de afrontamiento de los participantes del estudio. Como se mencionó anteriormente, la incertidumbre y la inestabilidad colaborarían con la elevación de los niveles de estrés y la mejor forma de acomodar cognitivamente esta realidad y este estrés sería el desentenderse de ellos hasta el momento en que por urgencia y criterio de realidad no quede otra posibilidad que la de afrontar directamente la situación. Si pudieran considerarse condiciones de vida previa y, empíricamente, el hecho de que estos jóvenes pertenecen principalmente a un nivel socioeconómico medio alto donde las circunstancias problemáticas más bien son afrontadas por los padres, permitiría entender los pocos recursos de afronte activo con los que estos jóvenes cuentan. Posteriormente, en la medida que vayan madurando y profundizando en sus estudios, podrían modular sus reacciones de estrés aún cuando todavía presenten cierta dificultad con las estrategias y estilos de afrontamiento utilizados frente a los problemas relacionados con el futuro y el sí mismo. Por ello también, la diferencia en relación con las estrategias más activas que utilizan cuando el problema se relaciona más con la universidad.

Es interesante ver como la variable evolutiva media en la relación estrés-afrontamiento. Así, encontramos que a mayor edad se observan menores niveles de estrés, lo que podría estar relacionado con la adquisición de mayores recursos de afrontamiento. Así, a medida que avanza la edad se utilizan más estrategias activas y focalizadas en el problema. Este hallazgo coincide con lo planteado por la literatura (Seiffge-Krenke, 1995). 
Lo que llama la atención es que, a mayor edad, se utiliza más la negación como estrategia de afrontamiento. En su definición, ésta consiste en rehusarse a creer que el estresor existe o tratar de actuar como si el estresor no fuera real (Carver, Scheier \& Weintraub, 1989). Esto podría deberse a que el estresor en su evaluación primaria es considerado inmanejable, o que esta asociación esté reflejando una de las características de la adolescencia en donde se tiende a pensar cosas como "a mí me va ir bien", "eso no me va a pasar a mî".

Finalmente, se realizó un análisis de regresión múltiple escalonado con el fin de determinar las principales variables que determinan los niveles de estrés, encontrándose que éstas pueden ser explicadas en un $20 \%$ por tres variables. El nivel de estrés está determinado por el grado de preocupación por el futuro, la edad y la utilización de la estrategia denominada supresión de actividades competentes. Esto reafirma lo antes señalado, a mayor edad, menores niveles de estrés. Asimismo, las manifestaciones de estrés disminuyen en la medida que se utiliza más la supresión de actividades competentes y es mayor cuanto más preocupación se percibe del futuro.

En resumen, podemos decir que esta población se caracteriza por presentar niveles moderados de estrés, predominando las reacciones emocionales del mismo. Se identificaron como principales preocupaciones aquellas relacionadas con el futuro y la visión del sí mismo, las que usualmente son afrontadas a través de la utilización de los estilos centrados en el problema y los centrados en la emoción, así como aquellas relacionadas con el uso de las estrategias más adaptativas y activas como son la reinterpretación positiva, la búsqueda de soporte social y la planificación. Es importante mencionar que también existe una notoria presencia del desentendimiento mental en el momento de afrontar aquellas preocupaciones asociadas con el futuro. Finalmente, las variables más determinantes de los niveles de estrés fueron el grado de preocupación por el futuro, la edad y la 
Estrés y afrontamiento en estudiantes de psicología

utilización de la estrategia denominada supresión de actividades competentes.

\section{Referencias}

Canessa, B. (2000). Adaptación de la prueba "escala de afrontamiento para adolescentes" en escolares entre 14 y 17 años de edad de diferente sexo y nivel socioeconómico. Tesis de licenciatura no publicada, Universidad de Lima, Lima.

Carver, C.H., Scheier, M. \& Weintraub, J. (1989). Assessing coping strategies: A theoretically based approach. Journal of Personality and Social Psychology, 56(2), 267-283.

Carver, C.H. \& Scheier, M. (1994). Situational coping and coping dispositions in a stressful transaction. Journal of Personality and Social Psychology, 66(1), 184-195.

Cassaretto, M., Oblitas, H. \& Valdéz, N. (2000). Ficha demográfica. Manuscrito no publicado.

Castro de la Mata, R. \& Rojas, M. (1998). Los jóvenes en el Perú: opiniones, actitudes y valores - 1997. Lima: Cedro.

Casuso, L. (1999). Adaptación de la Prueba COPE sobre estilos de afrontamiento en un grupo de estudiantes universitarios de Lima. Tesis de licenciatura no publicada, Pontificia Universidad Católica del Perú, Lima.

Chau, C. (1999). Consumo de bebidas alcohólicas en estudiantes universitarios: motivaciones y estilos de afrontamiento. Tesis de maestría no publicada, Pontificia Universidad Católica del Perú, Lima.

Chau, C. (2003). Determinantes del consumo de bebidas alcohólicas en estudiantes universitarios: el rol del estrés, expectivas y afrontamiento. Tesis doctoral no publicada, Universidad Católica de Lovaina, Lovaina, Bélgica.

Endler, N. \& Parker, J. (1994). Assessment of multidimensional coping: Task emotion and avoidance strategies. Psychological Assessment, 6 (1), 50-60. 
Fernández-Abascal, G., Palmero, F., Chóliz, M. \& Martínez, F. (1997). Cuaderno de prácticas de motivación y emoción. Madrid: Pirámide.

Folkman, S., Lazarus, R., Gruen, R. \& De Longis, A. (1986). Appraisal, coping, health status and psychological symptoms. Journal of Personality and Social Psychology, 50(3), 571-579.

Frydenberg, E. (1997). Adolescent coping: Theoretical and research perspective. Londres: Routledge.

Frydenberg, E. \& Lewis, R. (1993). Boys play sport and girls turn to others: Age, gender and ethnicity as determinants of coping. Journal of Adolescence, 16, 253-266.

Frydenberg, E. \& Lewis, R. (1997). Manual: escalas de afrontamiento para adolescentes (ACS). Madrid: TEA ediciones.

Frydenberg, E. \& Lewis, R. (1999). Things don't get better just because you're older: A case for facilitating reflection. British Journal of Educational Psychology, 69, 81-94.

Hallman, W. \& Wandersman, A. (1992). Attribution of responsability and individual and collective coping with environmental threats. Journal of Social Issues, 48(4), 101-118.

Herbert, T. (1994). Stress and the immune system. World Health, 47(2), 4-5.

Hernández, R., Fernández, C. \& Baptista, P. (1996). Metodología de la investigación. México: McGraw Hill.

Hobfoll, S. (1989). Conservation of resources: A new attempt at conceptualizing stress. American Psychologist, 44(3), 513-524.

Kavsek, M. \& Seiffge-Krenke, I. (1996). The differentiation of coping traits in adolescence. International Journal of Behavioral Development, 19 (3), 651-668.

Kobasa, S., Maddi, S. \& Kahn, S. (1982). Hardiness and health: A prospective study. Journal of Personality and Social Psychology, 42 (1), 168-177.

Lazarus, R. \& Folkman, S. (1986). Estrés y procesos cognitivos. Barcelona: Martínez Roca S.A.

Lepose, S., Erans, G. \& Schneider, M. (1991). Dynamic role of social support in the link between chronic stress and psychological 
distress. Journal of Personality and Social Psychology, 61(6), 899909.

Martínez, P. \& Morote, R. (2001). Preocupaciones de adolescentes de Lima y sus estilos de afrontamiento. Revista de Psicología de la Pontificia Universidad Católica del Perú, 19 (2), 211-236.

Montenegro, C. (2001). Adaptación del cuestionario de problemas y del cuestionario de afrontamiento a través de situaciones (CASQ) en escolares de primer y quinto año de secundaria de Lima. Tesis de licenciatura no publicada, Pontificia Universidad Católica del Perú, Lima.

Moreno, B., Oliver, C. \& Aragoneses, A. (1991). El burnout una forma específica-de estrés laboral. En G. Buela-Casal \& V. Caballo (Eds.), Manual de psicología clínica aplicada. Madrid: Siglo XXI.

Papalia, D. (1992). Desarrollo Humano. México: McGraw Hill.

Patterson, J. \& McCubbin, H. (1987). Adolescent coping style and behaviors: Conceptualization and measure. Journal of Adolescence, 10, 163-186.

Phelan, P., Yu, C. \& Davidson, A. (1994). Navigating the psychosocial pressures of adolescence. En L. Trahtemberg (1995), Tensión en los escolares adolescentes. Gerencia, XXV (220), 22-24.

Phelps, S. \& Jarvis, P. (1994). Coping in adolescence: Empirical evidence for a theoretically based approach to assessing coping. Journal of Youth and Adolescence, 23 (3), 359-371.

Ragúz, M. (1995). Construcciones sociales y psicológicas de mujer, hombre, feminidad, masculinidad y género en diversos grupos poblacionales. Lima: Pontificia Universidad Católica del Perú.

Rodríguez, J. (1995). Psicología de la salud. Madrid: Síntesis.

Rojas, R. (1997). Ansiedad, cólera y estilos de afrontamiento en portadores del VIH. Tesis de maestría no publicada, Pontificia Universidad Católica del Perú, Lima.

Russell, D., Booth, B., Reed, D. \& Laughlin, P. (1997) Personality, social networks and perceived social support among alcoholics: A structural equation analysis. Journal of Personality, 65 (3), 649-692. 
Sandín, B. (1995). El estrés. En A. Belloch, B. Sandín \& F. Ramos (Eds.), Manual de psicopatología. Madrid: McGraw Hill.

Seiffge-Krenke, I. (1995). Stress, coping and relationships in adolescence. Nueva Jersey: Lawrence Erlbaum Associates, Inc.

Seiffge-Krenke, I. (1998). Adolescents' health. A developmental perspective. Nueva Jersey: Lawrence Erlbaum Associates, Inc.

Shepperd, J. \& Kashani, J. (1991). The relationship of hardiness, gender and stress to health outcomes in adolescents. Journal of Personality, 59 (4), 747-768.

Stone, A., Greenberg, M., Kennedy-Moore, E. \& Newman, M. (1991). Self-report, situation-specific coping questionnaires: What are they measuring? Journal of Personality and Sócial Psychology, 61 (4), 648-658.

Taylor, S. (1999). Health psychology. Nueva York: McGraw Hill.

Valdez, N. (1999). Cuestionario de la Respuesta de Estrés. En N. Valdez, Estrés y recursos de afrontamiento en un grupo de adolescentes embarazadas. Tesis no publicada, Pontificia Universidad Católica del Perú, Lima.

Wiebe, D. (1991). Hardiness and stress moderation: A test of proposed mechanisms. Journal of Personality and Social Psychology, 60 (1), 89-99. 